\title{
Contribution of swimming, cycling and running in the final performance in different distances of triathlon races
}

\begin{abstract}
The aim of the study was to analyze which modality (swimming, cycling or running) may interfere significantly to the final performance of different triathlon events. The present study observed the last five results of Olympic Distance, Half Distance and Full Distance (Ironman) of World Championship from 2010 to 2015. The athlete's times were tabulated separate per: swimming time, cycling time, running time and total time. The sample was divided in two groups: (G1) athletes who were classified among the five best of each step and (G2) the athletes classified between sixth and tenth place. The total sample was composed by male and female athletes $(\mathrm{N}=300)$. Statistical analysis: the regression, correlation and comparison model was used between two groups for the three distances of triathlon tests and for all investigated variables. The results pointed that for each type of test and athlete one modality may be more deterministic to the final performance. Therefore, the training programs should consider that triathlon is one only modality; thus, the training planning should be focused in a unique way taking into account the specificity of race.
\end{abstract}

Volume I Issue 5 - 2017

\author{
Claudio Scorcine, ${ }^{1,2}$ Rodrigo Pereira, ${ }^{1,2}$ \\ Fabricio Madureira,' Emilson Colantonio \\ 'Universidade Metropolitana de Santos, Brazil \\ 2Universidade Federal de São Paulo, Brazil
}

\begin{abstract}
Correspondence: Claudio Scorcine, Universidade Metropolitana de Santos, Brazil,Tel 55I3997929|46, Email claudio-scorcine@uol.com.br
\end{abstract}

Received: October 25, 2017 | Published: November 16, 2017

Keywords: triathlon, swim, cycle, run, performance, olympic distance

\section{Introduction}

Triathlon is one of the fastest growing sports in the world and the estimates show that there are thousands of practitioners, qualified in the execution of three consecutive sports (swimming, cycling and running, respectively). ${ }^{1,2}$ There are different types of races, with different distances: short distance $(750 \mathrm{~m}, 20 \mathrm{~km}$ and $5 \mathrm{~km})$; Olympic distance $(1500 \mathrm{~m}, 40 \mathrm{~km}$ and $10 \mathrm{~km})$, half distance $(1900 \mathrm{~m}, 90 \mathrm{~km}$ and $21 \mathrm{~km})$ and full distance $(3800 \mathrm{~m}, 180 \mathrm{~km}$ and $42 \mathrm{~km})$. Analyzing the races is possible to describe that swimming ranges from 10 to $20 \%$ of the total, cycling between 50 and $60 \%$ and running 30 to $40 \%$ of the race. ${ }^{3}$ However, despite of cycling to be a higher percentage of execution in the race, researchers descript that this type of individual (triathlete) should be versatile and master in the three modalities; throughout his practice during the years, as features of each task into different metrics and their influence on the overall performance of the evidence changes. ${ }^{5}$

The three modalities executed in the triathlon have some characteristics of their own and in the swimming part, the draft (swim behind an individual) is allowed, which can save up to $40 \%$ of the athlete's energy. Also, some races allow the use of wetsuits, which may interfere in the athlete's physical effort, decreasing heart rate and lactate level for the same effort. ${ }^{6}$ Research indicates that this modality when performed in a submaximal intensity $(80 \%)$ the final result of the race may be better, given the intensity achieved in cycling, when performed in this way. ${ }^{7}$ In cycling there are different rules for different distances, in the case of the Olympic races it is permitted to use the vacuum reducing caloric expenditure and physical exertion significantly improving athlete's running performance, ${ }^{9}$ already for the longer distances this strategy is not permitted, causing all athletes to start the running in similar conditions of physical wear and tear The run is the last modality to be performed and researchers report that this running performance should be harmed by muscle stress resulting from the physical exertion of previous tasks. ${ }^{10}$

Various factors might influence on performance prediction, Gilinsky et al. ${ }^{11}$ pointed out that for the race, the best predictors were age and number of participations in previous editions. ${ }^{11}$ In addition to these factors, the particularities and strategies in swimming, cycling and running, in their different distances according to the races, can also trigger changes in athletes' performance. All these factors must be taken into account for the elaboration of the training periodization, with the purpose of a maximum performance in the competitions. Researchers suggest that by the proportions of a triathlon race the cycling and running are decisive for the final result. ${ }^{12}$ However, it does not seem clear in the literature how is important each modality for the final performance of different distances in the triathlon.

Therefore, the aim of this investigation was to analyze the contribution of each modality to the final performance of triathlon events in the Olympic distances, half distance and full distance for males and females athletes.

\section{Methods}

\section{Participants}

For Olympic distance analyses, was observed five steps of the World Olympic Triathlon 2015, analyzing the 10 best male athletes $(n=50)$ and 10 best female athletes $(n=50)$ of each race. For half distance, was observed the results of the Half Ironman World Championship from 2010 to 2015 , analyzing the 10 best male athletes $(n=50)$ and 10 best female athletes $(\mathrm{n}=50)$. For a full distance, was observed the results of the Ironman World Championship, in Hawaii, from 2010 to 2015 , analyzing the 10 best male athletes $(n=50)$ and 10 best female athletes $(\mathrm{n}=50)$. All athletes were divided into two groups: $\mathrm{G} 1$ five placed and G2 from sixth to the tenth place of the race 


\section{Protocol}

All analyzes were conducted based on the execution time of each modality (swimming, cycling and running) and the total time athletes. Furthermore, comparisons were made between all variables of G1 and G2.

\section{Statistical analysis}

For the regression and correlation analysis, was used the total number of athletes each competition, where in each step the top 10 were classified. To obtain the indicative of which modality has the potential to be more deterministic in the final result was used the Beta value obtained through the analysis of linear logistic regression. After the regression analysis, the correlation analysis was performed to observe which of the three modalities correlated better with the total time of the race. Soon after, the group was divided between G1 (first to fifth placed) and G2 (sixth to tenth place) for the comparisons. For the comparisons of the results, the normality test was applied and after the confirmation the Student's T test was used for independent samples for the comparison between the first fifth places and from the sixth to the tenth places for swimming, cycling, running stages and the total race time. The significance level was set to $\mathrm{p} \leq 0,05$.

\section{Results}

It was possible to observe that the total time of the first five places does not differ from the time of the sixth to the tenth place in Olympic triathlon (Table 1) and still, in the men's group the swimming presented the highest correlation and Beta values in comparison to the total time of the race, already for the women the cycling seems to be more determinant for the final result. There were significant differences in total time when compared to male and female G1 and G2, respectively (Table 2). The best variable correlation observed to the total time was cycling for both groups, however all modalities appear to interfere in the final result.

There were significant differences in comparison of the total time for male and female athletes between G1 and G2 groups (Table 3) and for male athletes the run seems to be the most determinant modality for the final performance, whereas for female athletes the cycling was the modality that better correlated with the final performance.

Table I Mean and standard deviation of male and female athlete's times for the Olympic Triathlon comparing GI (5 first) and G2 (6 to I0 placed) in the modalities: swimming (Swim); Cycling (Cycle); Run (Run) and total time. The regression indices (Beta) Correlation and p of the comparison between groups

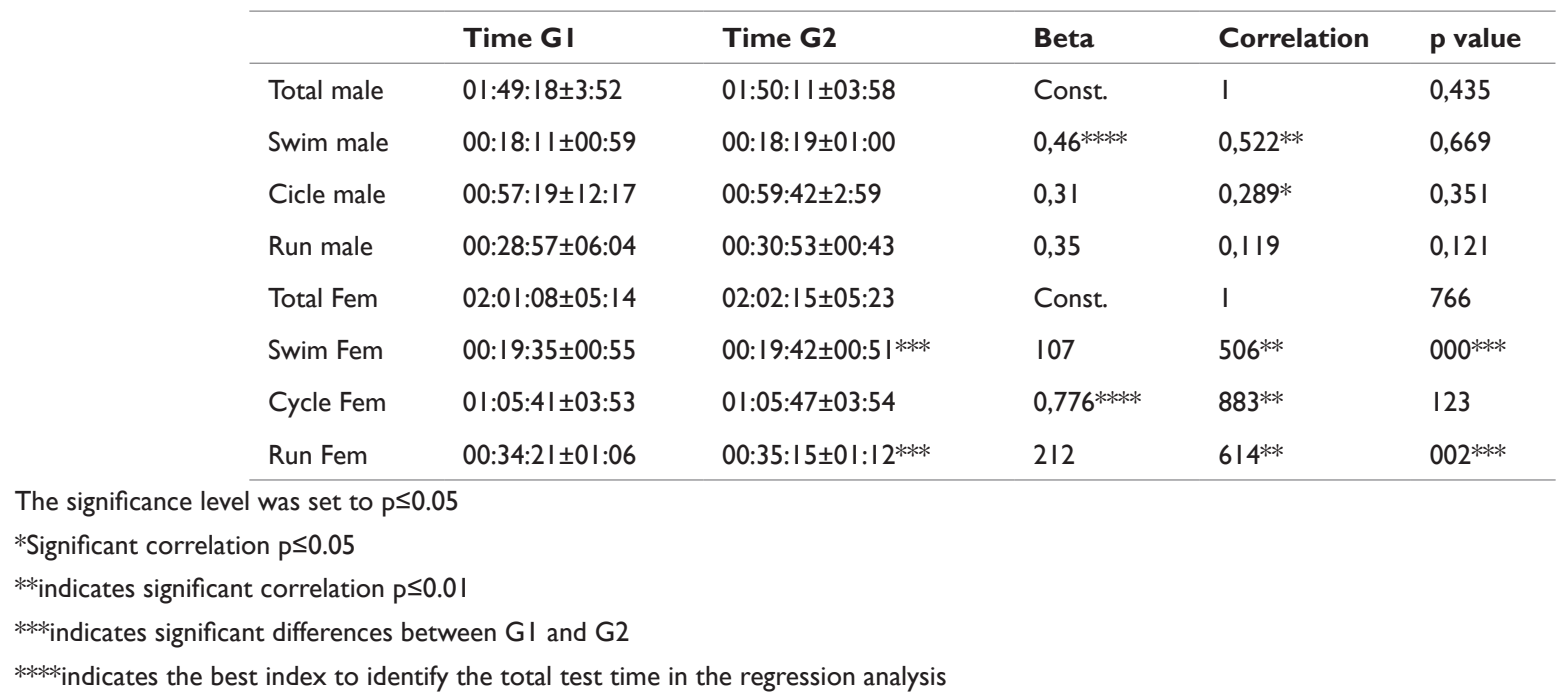

Table 2 Mean and standard deviation of male and female athlete's times for the half triathlon comparing GI (5 first) and G2 (6 to I0 placed) in the modalities: swimming (Swim); Cycling (Cycle); Run (Run) and total time. The regression indices (Beta) Correlation and p of the comparison between groups.

\begin{tabular}{llllll}
\hline & Time GI & Time G2 & Beta & Correlation & P value \\
\hline Total male & $03: 52: 10 \pm 06: 14$ & $03: 56: 22 \pm 06: 36^{* * *}$ & Const. & 1 & $014^{* * *}$ \\
Swim male & $00: 24: 07 \pm 01: 27$ & $00: 24: 09 \pm 01: 06$ & 194 & $354^{* *}$ & 911 \\
Cicle male & $02: 10: 14 \pm 04: 25$ & $02: 11: 29 \pm 04: 42$ & $0,667^{* * * *}$ & $898^{* *}$ & 288 \\
Run male & $01: 14: 19 \pm 02: 15$ & $01: 17: 06 \pm 03: 01 * * *$ & 456 & $725^{* *}$ & $000^{* * *}$ \\
Total Fem & $04: 24: 25 \pm 07: 27$ & $04: 30: 58 \pm 06: 46 * * *$ & Const. & 1 & $002^{* * *}$ \\
Swim Fem & $00: 27: 04 \pm 02: 30$ & $00: 26: 53 \pm 01: 38$ & 268 & $506 * *$ & 766 \\
Cycle Fem & $02: 27: 10 \pm 04: 57$ & $02: 32: 27 \pm 04: 36^{* * *}$ & $0,685^{* * * *}$ & $883^{* *}$ & $000^{* * *}$ \\
Run Fem & $01: 26: 14 \pm 03: 32$ & $01: 27: 39 \pm 02: 51$ & 420 & $614^{* *}$ & 123 \\
\hline
\end{tabular}

The significance level was set to $p \leq 0.05$.

*Significant correlation $\mathrm{p} \leq 0.05$

** indicates significant correlation $\mathrm{p} \leq 0.01$

*** indicates significant differences between GI and G2

*****indicates the best index to identify the total test time in the regression analysis 
Table 3 Mean and standard deviation of male and female athlete's times for the full triathlon comparing GI (5 first) and G2 (6 to I0 placed) in the modalities: swimming (Swim); Cycling (Cycle); Run (Run) and total time. The regression indices (Beta) Correlation and p of the comparison between groups

\begin{tabular}{llllll}
\hline & Time GI & Time G2 & Beta & Correlation & p value \\
\hline Total male & $08: 17: 56 \pm 05: 36$ & $08: 26: 51 \pm 04: 19$ & Const. & I & $0,000^{* * *}$ \\
Swim male & $00: 52: 10 \pm 01: 21$ & $00: 52: 20 \pm 01: 44$ & 240 & 0,189 &, 670 \\
Cicle male & $04: 30: 59 \pm 05: 01$ & $04: 34: 00 \pm 05: 52$ & 839 & $0,431^{*}$ & $0,036^{* * *}$ \\
Run male & $0250: 34 \pm 04: 35$ & $02: 56: 14 \pm 06: 40$ & $0,955^{* * * *}$ & $0,618^{* *}$ & $0,000^{* * *}$ \\
Total Fem & $09: 08: 48 \pm 08: 55$ & $09: 22: 19 \pm 07: 07$ & Const. & I & $0,000^{* * *}$ \\
Swim Fem & $00: 57: 15 \pm 03: 41$ & $01: 00: 16 \pm 05: 52$ & 492 & 0,283 & $0,021^{* * *}$ \\
Cycle Fem & $05: 01: 27 \pm 05: 58$ & $05: 07: 06 \pm 10: 42$ & $0,865 * * * *$ & $0,585^{* *}$ & $0,014^{* * *}$ \\
Run Fem & $03: 05: 19 \pm 07: 13$ & $03: 10: 05 \pm 07: 43$ & 749 & $0,466^{*}$ & $0,016^{* * *}$ \\
\hline
\end{tabular}

The significance level was set to $\mathrm{p} \leq 0.05$

*Significant correlation $\mathrm{p} \leq 0.05$

**indicates significant correlation $\mathrm{p} \leq 0.01$

***indicates significant differences between GI and G2

****indicates the best index to identify the total test time in the regression analysis

\section{Discussion}

Although swimming represents the lowest proportion of the race in the three distances investigated, for the male gender in the Olympic distance was the most determinant modality for the final result, however, when observed the first five (G1) and the sixth to the tenth placed (G2) there were no significant differences in any variable. For females, the swimming and running presented a good correlation with final performance, showing that there was a difference between G1 and G2 in these variables for the five steps investigated. In the half distance events it was possible to observe significant differences between G1 and G2 men for the total time of race and the time of run; however, the cycling had the best correlation with the final time, demonstrating that the fast athletes of this modality won the race. For the female gender, it was possible to observe significant differences in the total time and in the cycling modality and this detail should be the most determinant point for the athlete to be better classified in the race. With regard to the full distance, for the male athletes, it was possible to observe differences between the total time, cycling and running modality, when comparing G1 and G2 in the six stages of the World Championship; being the run the best modality correlated with the athlete final performance. For females, the results showed significant differences in all variables, with cycling being the most determinant modality for the final performance of the athlete.

Studies to determine predictors in triathletes performance it has been conducted Gilinsky et al. ${ }^{11}$ whom was possible observed that the best time of running modality was the better predictor for performance in the race and it should be influenced by age. In this same study, training time of cycling modality had no influence on the same task. ${ }^{11}$ In comparison between men and women as predictors of performance in the full distance triathlon (Ironman), the anthropometric variables appear to be determinant, analyzing the last 35 years. ${ }^{13}$ The running seems to be determinant in all races, with some inter and intragroup differences in the different triathlon distances. According to Etxebarria et al. ${ }^{14}$ some attributes used in cycling may influence in this modality, such as the perception of physiological responses. ${ }^{14}$ In general context of triathlon events of different distances, Olympic, half distance and full distance seems to have a distinct characteristics, demonstrating that there is no predominant modality in these three types of competition, being the most influential swimming in the shortest competitions and run in the longest distances for the males athletes, although some researchers indicate that a better time in this type of race is not due to the good swim time, however the prior physiological conditions can interfere in modality consequent $;{ }^{15}$ although some papers shown that the how smaller the distance of the race, the greater the influence of swimming on the other tasks. ${ }^{16}$

In relation to running, this modality may be affected according to distance and age of the athlete, which may influence final performance. ${ }^{17}$ Some studies demonstrate biomechanical changes of the race after cycling and this are directly associated to the muscle fatigue caused by the previous tasks. Cycling, even though it is not determinant in some distances for final result, is still the modality that the athlete spends the most time during the race and can influence the run modality as a significant way, therefore, the coaches must be careful about the type of the training they will prescribe during the season. Another study which investigated 14 athletes the aim was to observe the influence of high intensity training in the performance modifications. The authors observed improvements in mean power, decreases in heart rate, blood lactate and perceived exertion. ${ }^{14}$ The triathlon can be seen with a single modality and not as the sum of three distinct modalities ${ }^{18,19}$ and should not to be prioritize training in a single sport or modalities separate as swimming, cycling or running. Researchers have shown that triathlon is one only modality and the training planning should include the transitions that paramount to the good final performance..$^{20,21}$

\section{Acknowledgements}

None.

\section{Conflict interest}

There is no conflict of interest of any author of the article. 


\section{References}

1. Bernard T, Sultana F, Lepers R, et al. Age-related decline in Olympic triathlon performance: effect of locomotion mode. Exp Aging Res. 2009;36(1):64-78.

2. Cohen L. Triathlons and medicine: the race to catch up. CMAJ. 1986;134(8):938.

3. Galy O, Manetta J, Coste O, et al. Maximal oxygen uptake and power of lower limbs during a competitive season in triathletes. Scand J Med Sci Sports; 2003;13(3):185-193.

4. Laursen PB, Rhodes EC. Factors affecting performance in an ultraendurance triathlon. Sports Med. 2001;31(3):195-209.

5. Figueiredo P, Marques EA, Lepers R. Changes in Contributions of Swim, Cycle and Run Performances on Overall Triathlon Performance over a 26-year period. J Strength Cond Res. 2016;30(9):2406-2415.

6. Delextrat A, Bernard T, Hausswirth C, et al. Wetsuits and energy output by cyclists after swimming. Canadian Journal of Applied PhysiologyRevue Canadienne De Physiologie Appliquee. 2003;28(3):356-369.

7. Peeling PD, Bishop DJ, Landers GJ. Effect of swimming intensity on subsequent cycling and overall triathlon performance. Br J Sports Med 2005;39(12):960-964.

8. Belloli M, Giappino S, Robustelli F, et al. Drafting effect in cycling: Investigation by wind tunnel tests. Procedia Engineering. 2016;147:38-43.

9. Hausswirth C, Vallier JM, Lehenaff D, et al. Effect of two drafting modalities in cycling on running performance. Med Sci Sports Exerc. 2001;33(3):485-492.

10. Gottschall JS, Palmer BM. The acute effects of prior cycling cadence on running performance and kinematics. Medicine and science in sports and exercise. 2002;34(9):1518-1522.
11. Gilinsky N, Hawkins KR, Tokar TN, et al. Predictive variables for halfIronman triathlon performance. J Sci Med Sport. 2014;17(3):300-305.

12. Vleck VE, Bürgi A, Bentley DJ. The consequences of swim, cycle, and run performance on overall result in elite olympic distance triathlon. Int J Sports Med. 2006;27(1):43-48.

13. Knechtle B, Knechtle R, Stiefel M, et al. Variables that influence Ironman triathlon performance - what changed in the last 35 years? Open Access J Sports Med. 2015;6:277.

14. Etxebarria N, Anson JM, Pyne DB, et al. High-intensity cycle interval training improves cycling and running performance in triathletes. Eur $J$ Sport Sci. 2014;14(6):521-529.

15. Margaritis I. Facteurs limitants de la performance en triathlon. Canadian Journal of Applied Physiology. 1996;21(1):1-15.

16. Neto SLV, Smirmaul BDPC, Pignata BH, et al. Efeito do nadar sobre o desempenho do pedal e corrida no triathlon super-sprint. Rev educ fis. 2014;25(1):45-51.

17. Wu SS, Peiffer JJ, Brisswalter J, et al. Factors influencing pacing in triathlon. Open Access J Sports Med. 2014;5:223-234.

18. Hue O, Gallais LD, Prefaut C. Specific pulmonary responses during the cycle-run succession in triathletes. Scand J Med Sci Sports. 2001;11(6):355-361.

19. Sleivert G, Rowlands D. Fatores fisiológicos associados ao sucesso no triatlo. Rev Sprint Rio de Janeiro. 2000;(107):4-14.

20. Millet GP, Vleck VE. Physiological and biomechanical adaptations to the cycle to run transition in Olympic triathlon: review and practical recommendations for training. Br J Sports Med. 2000;34(5):384-390.

21. Ribeiro LFP, Galdino R, Balikian P. Resposta lactacidêmica de nadadores e triatletas em função da utilização de "esteira" durante natação em velocidade correspondente ao limiar anaeróbio. Rev Paul Educ Fís. 2001;15(1):55-62. 